\title{
POTENTIAL ENERGY FLUCTUATION IN QUASI TWO-DIMENSIONAL ELECTRON GAS DUE TO EXTERNAL IONIZED IMPURITIES*
}

\author{
P. KĘBLIŃSKI \\ Institute of Physics, Polish Academy of Science \\ Al. Lotników 32/46, 02-668 Warszawa, Poland
}

\begin{abstract}
Two-dimensional electron gas separated from Coulomb centers by a spacer is considered. Electrons are treated semi classically in limit of weak fluctuation and $T=0$. Two screening models, Thomas-Fermi and RPA, are independently used. The second moment of the potential energy distribution is calculated analytically as a function of the spacer width. The qualitative arguments for the Gaussian character of the distribution are given and the density of states for such a distribution is calculated.
\end{abstract}

PACS numbers: $73.20 . \mathrm{Hb}$

\section{Screening models}

We want to find the response of the two-dimensional electron gas to a point charge. The electron gas is two-dimensional in this sense that its density can be represented by the Dirac function $\delta(z)$, where $z$ is the coordinate perpendicular to the plane. It was shown that with such a model (see [2]) one can still use the effective mass approximation and the energy measured from the bottom of the conduction band can be represented by

$$
E=\frac{\hbar^{2} k^{2}}{2 m}
$$

where $k$ is the two-dimensional wave vector, $m$ - the effective mass. It gives the energetic density of states:

$$
g(\varepsilon)=\frac{m}{\pi \hbar^{2}}
$$

*This work was partially supported by the Project G-MEN-129/90. 
and the Fermi level:

$$
E_{\mathrm{F}}=\frac{N_{\mathrm{s}} \pi \hbar^{2}}{m},
$$

where $N_{\mathbf{s}}$ is the surface electron density. In the limit of weak fluctuations the Thomas-Fermi approach can be used to find a response for a single center located at the point $\left(0,0, z_{0}\right)$. The Poisson equation can be linearized and the value of the screened potential in $z=0$ plane is given by [3]

$$
\Phi_{\mathrm{TF}}\left(r, z_{0}\right)=\frac{e}{\kappa} \int_{0}^{\infty} \frac{q \exp \left(-q z_{0}\right)}{q+q \mathrm{TF}} J_{0}(q r) \mathrm{d} q,
$$

where $\kappa$ is the dielectric constant, $q_{\mathrm{TF}}=2 / a_{\mathrm{B}}, a_{\mathrm{B}}$ - the effective Bohr radius, $J_{0}$ - the zero order Bessel function and $r^{2}=y^{2}+x^{2}$. We can treat $q_{\mathrm{TF}}$ as the two-dimensional screening parameter, note that in $2 \mathrm{D} q_{\mathrm{TF}}$ is independent on the electron concentration. The same formula for the potential can be obtained in RPA [4] but with $q_{\mathrm{TF}}$ replaced by $q_{\mathrm{RPA}}$ :

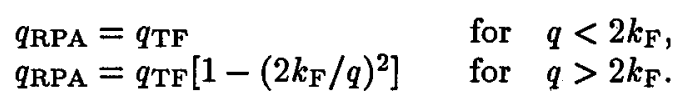

\section{Fluctuations}

We consider Coulomb centers randomly distributed in the region between $z_{-}$and $z_{+}\left(z_{+}>z_{-}>0\right)$ acting on the electron plane $(z=0)$. The doping is disordered so that the density correlation function is given by a formula:

$$
\left\langle\rho(\boldsymbol{r}), \rho\left(\boldsymbol{r}^{\prime}\right)\right\rangle=N_{V} \delta\left(\boldsymbol{r}-\boldsymbol{r}^{\prime}\right)
$$

$N_{V}$ - the average doping density. Potential can be obtained using Green functions:

$$
\phi(r)=\int G\left(r, r^{\prime}\right) \rho\left(r^{\prime}\right) \mathrm{d}^{3} r^{\prime}
$$

so we can calculate the second moment of the potential as the value of the potential correlation function for $r=r^{\prime}$. We are still interested in potential at $z=0$ so from (1.3) and (2.1)

$$
\left\langle\phi^{2}\right\rangle=N_{V} \int G^{2}(r) \mathrm{d}^{3} r
$$

where the integration is over the doping area and

$$
G(r)=\frac{e}{\kappa} \int_{0}^{\infty} \frac{q \exp (-q z)}{q+q} J_{0}(q r) \mathrm{d} q .
$$

In the last formula $q=q_{\mathrm{TF}}$ or $q=q_{\mathrm{RPA}}$. Equations (2.3) and (2.4) give 


$$
\begin{aligned}
& \left\langle\phi^{2}\right\rangle=\frac{2 \pi N_{V} e^{2}}{\kappa^{2}} \int_{z_{-}}^{z}=\mathrm{d} z \int_{0}^{\infty} \mathrm{d} r \int_{0}^{\infty} \mathrm{d} q \int_{0}^{\infty} \mathrm{d} q^{\prime} \frac{q q^{\prime} \exp \left[-\left(q+q^{\prime}\right) z\right]}{(q+q)\left(q^{\prime}+q\right)} \\
& \times J_{0}(q r) J_{0}\left(q^{\prime} r\right) r,
\end{aligned}
$$

where the integration over the angular variable is already performed. The integration over $r$ gives $\delta\left(q-q^{\prime}\right)$, so that after the integration over $q^{\prime}$ we obtain the final formula:

$$
\gamma^{2}=e^{2}\left\langle\phi^{2}\right\rangle=\frac{2 \pi N_{V} e^{4}}{k^{2}} \int_{z_{-}}^{z_{+}} \mathrm{d} z \int_{0}^{\infty} \mathrm{d} q \frac{q \exp (-2 q z)}{(q+\bar{q})^{2}},
$$

where $\gamma$ is the potential energy dispersion. The values of $\gamma$, in case of the planar doping $\left\{z_{+} \rightarrow z_{-} \rightarrow x_{0},\left(z_{+}-z_{-}\right) N_{V} \rightarrow N_{\mathrm{d}}\right\}$, are presented in Fig. 1 for GaAs parameters. In the region of the spacer width $25 \AA<x_{0}<200 \AA$, where the

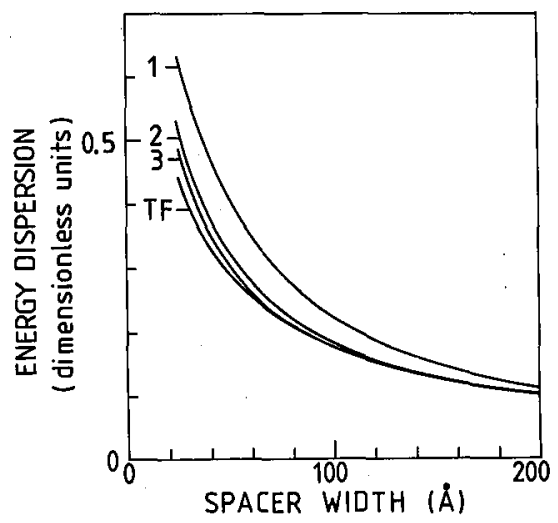

Fig. 1. The ratio of the dispersion of the potential energy fluctuations to the square root of the doping density as a function of the spacer width (doping in a plane, electron density in $\mathrm{cm}^{-2}$ ). Curves (1), (2), (3) present RPA results for various values of $N_{\mathrm{e}}$ : $10^{10} \mathrm{~cm}^{-2}(1), 5 \times 10^{10} \mathrm{~cm}^{-2}(2), 10^{11} \mathrm{~cm}^{-2}(3)$.

potential energy fluctuations are significant compared to the Fermi energy (e.g. $\gamma / E_{\mathrm{F}}=1 / 8$ for $N_{\mathrm{e}}=N_{\mathrm{d}}=10^{12} \mathrm{~cm}^{-2}$ and $x_{0}=100 \AA$ ), the dispersion $\gamma$ decrease with increasing $x_{0}$ roughly as $1 / x_{0}$. Compared to the RPA results T-F approach overestimates the screening for low electron densities, the relative difference is about $10 \%$ at $N_{\mathrm{e}}=10^{10}\left(\mathrm{~cm}^{-2}\right)$ and practically vanish at $N_{\mathrm{e}}=10^{12}$.

\section{Distribution function and density of states}

When the potential at a given point at the electron plane originates effectively from several impurities or more the Poisson distribution of impurities can be replaced by the Gaussian $[2,5]$. The larger the doping concentration and the wider the spacer the replacement is better justified. As a consequence within the linear 
screening theory the odd moments of the potential energy distribution vanish, the even moments reduce to powers of the second moment, so that the potential energy distribution is also Gaussian:

$$
P(V)=\frac{1}{(2 \pi)^{0.5} \gamma} \exp \left(-V / 2 \gamma^{2}\right)
$$

Now we can introduce local density of states

$$
\begin{array}{llll}
g(\varepsilon, r)=m / \pi \hbar^{2} & \text { for } & \varepsilon>V(r), \\
g(\bar{\varepsilon}, r)=0 & \text { for } & \varepsilon<V(r) .
\end{array}
$$

The global density of states is proportional to the fraction of the surface, where the energy is higher than $\varepsilon$

$$
g(\varepsilon)=\frac{m}{\pi \hbar^{2} L^{2}} \int_{\varepsilon>V(x, y)} \mathrm{d} x \mathrm{~d} y,
$$

where $L^{2}$ is the total area. From (3.1) and (3.3) we obtain finally

$$
g(\varepsilon)=\frac{m}{\pi \hbar^{2}(2 \pi)^{0.5} \gamma} \int_{-\infty}^{\varepsilon} \exp \left(-V^{2} / 2 \gamma^{2}\right) \mathrm{d} V .
$$

For $\varepsilon<0$ we have got the tail with the non-zero density of states given by error function.

\section{References}

[1] B.J. Shklovskij, A.L. Efros, Electronic Properties of Heavy Doped Semiconductors, Nauka, Moscow 1979.

[2] T. Ando, A.B. Fowler, F. Stern, Rev. Mod.'Phys. 54, 437 (1982).

[3] F. Stern, Phys. Rev. Lett. 18, 546 (1967).

[4] P.F Maldague, Surface Sci. 73, 296 (1978).

[5] T.N. Morgan, Phys. Rev. 139, 1A, 343 (1965). 